\title{
On the Slow Roll Expansion for Brane Inflation
}

\author{
Michat Spaliński * \\ Sottan Institute for Nuclear Studies \\ ul. Hoża 69, 00-681 Warszawa, Polska.
}

\begin{abstract}
One possibility for identifying the inflaton in the framework of string theory is that it is a $D$-brane modulus. This option involves a specific, non-canonical form of the kinetic energy - the Dirac-Born-Infeld action. This note investigates the applicability of the slow roll approximation in inflationary models of this type. To this end the slow roll expansion of Liddle, Parsons and Barrow is derived for the case of the DBI action. The resulting slow roll conditions augment the standard ones valid in the case of canonical kinetic terms. It is also shown that in DBI models inflation does not require that the potential dominate the energy density.
\end{abstract}

KEYWORDS: string cosmology.

*Email: mspal@fuw.edu.pl 


\section{Contents}

1. Introduction 1

2. The slow roll expansion in the canonical case 3

3. The slow roll expansion for DBI inflation 6

4. Corrections to inflationary observables 9

5. Conclusions 11

\section{Introduction}

The success of inflationary cosmology makes it important to look for its realization in string theory. In particular, it is important to understand what possibilities exist for identifying the degrees of freedom which could lead to an inflationary effective field theory at energies below the Planck scale. For a long time geometric closed string moduli were considered to be possible inflaton candidates. Recently [1] the possibility that the inflaton might be an open string modulus ${ }^{1}$ has been investigated in a number of papers (for reviews and references see for example [2, 3, 5, 4]). This option is interesting and novel in a number of ways. One important aspect of this idea is that the kinetic terms for the inflaton are non-canonical [6, 7] and are uniquely determined to all orders in $\alpha^{\prime}$ by the Dirac-Born-Infeld action [8]. It is thus a very distinctive consequence of identifying the inflaton with an open string mode. The DBI kinetic terms involve higher than quadratic powers of the time derivative of the

\footnotetext{
${ }^{1}$ Once supersymmetry is broken this open string mode is no longer a modulus.
} 
inflaton and it is important to determine whether these nonlinearities have practically measurable consequences.

Non-canonical kinetic terms of various degrees of generality have been the focus of interest for some time [9]-13]. Here however the form of the kinetic energy density is determined by well motivated string computations. The specific string models which have been considered in this context involve a $D$-brane approaching an anti- $D$-brane lodged at the bottom of a throat [14, 15, 16, 17, 18, 19] in a warped Calabi-Yau compactification [20]. While leaving many issues still unresolved at this point in time, it is clear that this type of model represents a fairly generic situation in string theory. The stringy geometry at the heart of this scenario is encoded at the effective field theory level in two objects: the usual potential energy density $V$ and another function of the inflaton field denoted below by $f$. This latter object is related to the geometry of the throat in the compactification manifold.

It was pointed out in [6] that the DBI action accounts for a speed limit on moduli space. This arises because the kinetic energy density depends on the time derivative of the inflaton via a factor $\gamma$, which is unity at small velocity and grows without bound as the speed limit is reached. Attention has mostly focused on the case when this limit is attained [6, 0, 16, 17]. The term DBI inflation has been used mostly to refer to this case. Here this term will be used more generally to refer to inflation in models with Dirac-Born-Infeld kinetic terms even when far from the speed limit. This note is devoted to the more mundane situation when $\gamma$ is close to unity, i.e. when the DBI action only gives small corrections to the standard results. This is potentially relevant since it looks like models with large $\gamma$ may have difficulties accommodating limits on nongaussianity and the primordial perturbations 18, 21, 22, 19.

The slow-roll approximation [23], developed in the context of effective field theory models with canonical kinetic terms, places flatness constraints on the potential energy density appearing in the effective field theory action. In the DBI case the action besides the potential also involves the function $f$ mentioned above, so it is interesting to ask what properties must this function possess in order that the slow roll approximation be valid. This question is addressed here by studying the slow roll expansion of Liddle, Parsons and Barrow [24], suitably generalized to the case of 
DBI kinetic terms. The lowest order in this expansion is the slow roll approximation; requiring that corrections to the leading order be small provides constraints on the effective action. In the canonical case this type of analysis implies the well-known conditions of the "potential slow roll" parameters $\epsilon_{V}$ and $\eta_{V}$. In the case of DiracBorn-Infeld models it turns out that in addition to these parameters one also needs to consider the dimensionless quantity $f V$ which enters the slow roll expansion at second non-leading order (which is then the usual $\eta_{V}$ parameter first appears). The generalized slow roll conditions involve the sum of $\eta_{V}$ and $\epsilon_{V} f V$, so barring a cancellation the standard slow roll conditions need to be augmented by the condition $\left|\epsilon_{V} f V\right| \ll 1$, which in the slow roll approximation is equivalent to $\gamma \approx 1$.

This note is organized as follows: section 1 reviews some aspects of the case of canonical kinetic terms and presents a simple iterative method for deriving the slow roll expansion, which easily generalizes to DBI models. This generalization is presented in section 3, where some other properties of DBI models are also discussed. In particular, it is shown that in contrast with the canonical case, in the "ultrarelativistic" limit DBI models do not require that the energy density be dominated by the potential for inflation to take place. In section ta number of physically relevant quantities related to the power spectra of primordial perturbations are calculated, including the small corrections arising from the DBI kinetic terms. These results are compared to the corresponding quantities computed in the "ultra-relativistic" regime. Finally, section 5 offers some closing remarks.

\section{The slow roll expansion in the canonical case}

To introduce notation, this section reviews the slow roll expansion in the case of a canonical kinetic term. The effective action for the inflaton is then of the form

$$
S=-\int d^{4} x \sqrt{-g}\left(\frac{1}{2}(\partial \phi)^{2}+V(\phi)\right) .
$$

For spatially homogeneous field configurations this leads to field equations for a perfect fluid with

$$
p=\frac{1}{2} \dot{\phi}^{2}-V(\phi)
$$




$$
\rho=\frac{1}{2} \dot{\phi}^{2}+V(\phi)
$$

The Einstein equations reduce to

$$
\begin{aligned}
\dot{\rho} & =-3 H(p+\rho) \\
3 M_{P}^{2} H^{2} & =\rho,
\end{aligned}
$$

where $M_{P}$ is the reduced Planck mass $\left(M_{P}^{2}=1 / 8 \pi G\right)$, the dot indicates a time derivative and $H \equiv \dot{a} / a$.

It is convenient to write these equations in first order form [25, 26, 27, 28], treating $\phi$ as the evolution parameter in place of $t$. From (2.2) - (2.5) it follows that

$$
\dot{\phi}=-2 M_{P}^{2} H^{\prime}(\phi)
$$

where the prime denotes a derivative with respect to $\phi$. Using this in (2.5) gives

$$
3 M_{P}^{2} H^{2}-V=2 M_{P}^{4} H^{2}
$$

This is the Hamilton-Jacobi form of the field equations [25, 26, 27, 28].

Given a potential density $V(\phi)$ one would like to solve the nonlinear equation (2.7) for $H(\phi)$, but this is very difficult to do in most cases. However, if one is interested in an inflating trajectory then there is a systematic expansion which can be carried out analytically, in principle to any order. To formulate it one needs to have an indication of which trajectories are inflationary. To this end one defines

$$
\epsilon_{H}=-\frac{\dot{H}}{H^{2}}
$$

which satisfies

$$
\frac{\ddot{a}}{a}=H^{2}\left(1-\epsilon_{H}\right)
$$

so that inflation occurs if and only if $\epsilon_{H}<1$. In terms of $H(\phi)$ one finds that

$$
\epsilon_{H}=2 M_{P}^{2}\left(\frac{H^{\prime}}{H}\right)^{2}
$$

It is sometimes convenient to express the condition $\epsilon_{H}<1$ in terms of the ratio $\epsilon$ of kinetic energy density to potential energy density defined by the equation:

$$
H^{2}=\frac{1}{3 M_{P}^{2}} V(1+\epsilon) \text {. }
$$


Using (2.7) it is easy to show that

$$
\epsilon_{H}=\frac{3 \epsilon}{1+\epsilon} .
$$

Using this the condition for inflation reads $\epsilon<1 / 2$, which is sometimes stated as the requirement that during inflation the potential should dominate the energy density. It will be shown below that in the case of DBI kinetic terms this is no longer the case.

The slow roll expansion of Liddle, Parsons and Barrow [24] (which extended the earlier work of Salopek and Bond [27]) is based on the observation that an inflating trajectory has $\epsilon_{H}<1$, which means that the right hand side of (2.7) is small relative to the terms on the left hand side of that equation. For this to be effective it is essential that the solution attains a late-time attractor [24]. The ensuing expansion may be derived in a number of ways. The method presented here has the benefit of being very simple to compute in an iterative way. Since it is clear that the expansion is one in the number of derivatives, one may introduce a parameter $\alpha$ which counts derivatives by replacing (2.7) with

$$
3 M_{P}^{2} H^{2}-V=\alpha M_{P}^{4} H^{\prime 2},
$$

and solving this equation in powers of $\alpha$ :

$$
H=\sum_{n} \alpha^{n} H_{n}
$$

At the end one is to set $\alpha=2$. Continuing up to second order in $\alpha$ as above leads to:

$$
\begin{aligned}
3 M_{P}^{2} H_{0}^{2}-V & =0 \\
2 H_{0} H_{1} & =M_{P}^{2} H_{0}^{\prime 2} \\
H_{1}^{2}+2 H_{0} H_{2} & =2 M_{P}^{2} H_{1}^{\prime} H_{0}^{\prime} .
\end{aligned}
$$

It is trivial to write down higher orders; proceeding up the second order as above leads to

$$
H(\phi) \simeq \sqrt{\frac{V(\phi)}{3 M_{P}^{2}}}\left\{1+\frac{1}{2} \epsilon_{V}+\epsilon_{V}\left(\eta_{V}-\frac{13}{8} \epsilon_{V}\right)\right\},
$$


where

$$
\begin{aligned}
\epsilon_{V} & =\frac{M_{P}^{2}}{6}\left(\frac{V^{\prime}}{V}\right)^{2} \\
\eta_{V} & =\frac{M_{P}^{2}}{3} \frac{V^{\prime \prime}}{V}
\end{aligned}
$$

are the standard "potential slow roll" (PSR) parameters ${ }^{2}$. With this normalization the slow roll expressions for the Hubble slow roll parameter and the ratio of kinetic to potential energy are:

$$
\epsilon_{H} \simeq 3 \epsilon_{V}, \quad \epsilon \simeq \epsilon_{V}
$$

Equation (2.18) coincides $^{3}$ with the expansion presented by Liddle, Parsons and Barrow [24]. It shows that the validity of this expansion rests on the smallness of $\epsilon_{V}$ as well as $\eta_{V}$.

A number of important calculations in the cosmology literature have been carried out in the Hubble slow roll approximation, which uses $\epsilon_{H}$ and its analogues expressed in terms of higher derivatives of $H(\phi)$. However, if one wishes to make contact with an effective field theory defined by a lagrangian, it is necessary to be able to calculate $H(\phi)$ in terms of the potential and any other objects and parameters appearing in the action. For this purpose the expansion (2.18) is very effective when applicable. The PSR parameters (2.19) are convenient, since they can be calculated immediately for any given model.

\section{The slow roll expansion for DBI inflation}

The effective action for the inflaton is the Dirac-Born-Infeld action, which for spatially homogeneous inflaton configurations takes the form [6, 18]

$$
S=-\int d^{4} x a(t)^{3}\left\{f(\phi)^{-1}\left(\sqrt{1-f(\phi) \dot{\phi}^{2}}-1\right)+V(\phi)\right\} .
$$

The function $f$ appearing here can be expressed in terms of the warp factor in the metric and the D3-brane tension. A number of specific models of this kind have been

\footnotetext{
${ }^{2}$ The normalization adopted here differs by a factor of $1 / 3$ from what is most commonly used.

${ }^{3}$ Apart from the different normalization of $\epsilon_{V}$ and $\eta_{V}$ the formula in [24] is given for $H^{2}$ rather than $H$.
} 
studied [7, 16, 17, 19, 29], mostly assuming an $A d S_{5}$ throat $\left(f \sim 1 / \phi^{4}\right)$ and various forms of the potential $V$. While this note does not consider specific examples, one should keep in mind that a number of models of this type are of interest.

The action (3.1) leads to field equations (2.4), (2.5) for a perfect fluid with

$$
\begin{aligned}
& p=\frac{\gamma-1}{f \gamma}-V(\phi) \\
& \rho=\frac{\gamma-1}{f}+V(\phi),
\end{aligned}
$$

where

$$
\gamma=\frac{1}{\sqrt{1-f(\phi) \dot{\phi}^{2}}} .
$$

To rewrite the field equations in Hamilton-Jacobi form one proceeds as in the canonical case. From (2.4)-(2.5) it follows that in this case that

$$
\dot{\phi}=-\frac{2 M_{P}^{2}}{\gamma} H^{\prime}(\phi) .
$$

This equation can easily be solved for $\dot{\phi}$ which allows one to express $\gamma$ as a function of $\phi$ :

$$
\gamma=\sqrt{1+4 M_{P}^{4} f H^{\prime 2}}
$$

Using this in 2.5) gives

$$
3 M_{P}^{2} H^{2}-V=\frac{\gamma-1}{f} .
$$

This is the Hamilton-Jacobi equation for DBI inflation [6]. Solving this equation for $H(\phi)$ given $V(\phi)$ and $f(\phi)$ is rather difficult. Most studies have considered the "ultra-relativistic" case $\gamma \gg 1$, where (3.7) simplifies considerably [6]. It is however clear that one can also implement the slow roll expansion here.

To do this, one needs to quantify under what conditions inflation takes place for this system. As discussed in section 2, in general inflation is taking place ${ }^{4}$ whenever $\epsilon_{H}<1$, where $\epsilon_{H}$ is defined by eq. (2.8). In the case of DBI inflation one finds (using (3.5)) that

$$
\epsilon_{H}=\frac{2 M_{P}^{2}}{\gamma}\left(\frac{H^{\prime}}{H}\right)^{2}
$$

\footnotetext{
${ }^{4}$ Of course for cosmology it is important that inflation takes place for long enough to generate adequate expansion.
} 
Defining the ratio $\epsilon$ of kinetic energy density to potential energy density as before one finds in the DBI case that

$$
\epsilon_{H}=\frac{\gamma+1}{2 \gamma} \frac{3 \epsilon}{1+\epsilon}
$$

At $\gamma$ close to unity this reduces to the canonical result, but for large gamma the additional factor in the above tends to $\frac{1}{2}$. The consequence of this is that the condition $\epsilon_{H}<1$ requires only $\epsilon<2$ : in this case the potential does not need to dominate the energy density for the system to inflate, unlike the case of canonical kinetic terms, when inflation requires $\epsilon<1 / 2$.

For the purpose of determining the slow roll expansion it will be convenient to work with the square of the Hamilton-Jacobi equation (3.10):

$$
\left(3 M_{P}^{2} H^{2}-V\right)\left\{1+\frac{1}{2} f\left(3 M_{P}^{2} H^{2}-V\right)\right\}=2 M_{P}^{4} H^{\prime 2}
$$

One may now proceed as in the canonical case. For an inflating trajectory on can treat the right hand side of $(3.10)$ as small relative to the other terms. Introducing the derivative-counting parameter $\alpha$ as before and looking for the solution in the form (2.14) one obtains, to second order,

$$
\begin{aligned}
\left(3 M_{P}^{2} H_{0}-V\right)\left\{1+\frac{1}{2} f\left(3 M_{P}^{2} H_{0}-V\right)\right\} & =0 \\
2 H_{0} H_{1} & =M_{P}^{2} H_{0}^{\prime 2} \\
\left(1+6 M_{P}^{2} f H_{0}^{2}\right) H_{1}^{2}+2 H_{0} H_{2} & =2 M_{P}^{2} H_{1}^{\prime} H_{0}^{\prime} .
\end{aligned}
$$

This yields

$$
H(\phi) \simeq \sqrt{\frac{V(\phi)}{3 M_{P}^{2}}}\left\{1+\frac{1}{2} \epsilon_{V}+\epsilon_{V}\left(\eta_{V}-\epsilon_{V}\left(\frac{13}{8}+\frac{1}{4} f V\right)\right)\right\} .
$$

The second order term in the above expression shows that the applicability of the slow roll approximation in this case requires that

$$
\left|\eta_{V}-\epsilon_{V}\left(\frac{13}{8}+\frac{1}{4} f V\right)\right| \ll 1
$$

in addition to the usual $\epsilon_{V}<1$. While a priori it could happen that the two contributions in (3.15) partially cancel, generically one needs

$$
\left|\eta_{V}\right| \ll 1, \quad\left|\epsilon_{V} f V\right| \ll 1 .
$$


To understand the meaning of the dimensionless quantity $f V$, note that the HamiltonJacobi equation (3.7) can be written as $\gamma=1+\epsilon f V$. If slow roll is valid one has $\epsilon \simeq \epsilon_{V}$ and so

$$
\gamma \simeq 1+\epsilon_{V} f V .
$$

Thus the second condition in (3.16) quantifies the expectation that $\gamma$ has to be close to unity for the slow roll approximation to apply.

\section{Corrections to inflationary observables}

This section looks at various physical quantities of interest in the slow roll approximation and presents leading corrections to these quantities arising due to the noncanonical kinetic terms. The slow roll results are compared with the corresponding "ultra-relativistic" results obtained in the limit of large $\gamma$.

The leading effect of DBI kinetic terms are corrections of order $\epsilon_{V}^{2} f V$. In some models, where $\epsilon_{V} f V$ is small but $f V$ is large these contributions would be larger than second order corrections of order $\epsilon_{V}^{2}$ and so on.

The basic quantity is the number of e-folds, which is given by

$$
N=\int d t H=\sqrt{\frac{3}{2}} \int \frac{d \phi}{M_{P}} \sqrt{\frac{\gamma}{\epsilon_{H}}} .
$$

Before considering the slow roll approximation it is convenient to write this in terms of $\epsilon$ using (3.9):

$$
N=\frac{1}{\sqrt{3}} \int \frac{d \phi}{M_{P}} \frac{\gamma}{\sqrt{\gamma+1}} \sqrt{\frac{1+\epsilon}{\epsilon}} .
$$

In the case of slow roll one has (3.17) which in leading order yields

$$
N=\int \frac{d \phi}{M_{P}} \frac{1}{\sqrt{6 \epsilon_{V}}}\left(1+\epsilon_{V}+\frac{3}{4} \epsilon_{V} f V\right) .
$$

The correction works to increase the number of e-folds relative to the leading slow roll result. For comparison, at large $\gamma$ one has

$$
\gamma \simeq \epsilon f V \gg 1
$$

so (4.2) gives

$$
N=\frac{1}{\sqrt{3}} \int \frac{d \phi}{M_{P}} \sqrt{f V(1+\epsilon)} .
$$


If one assumes that potential energy dominates the energy density (i.e. that $\epsilon \ll 1$ ) this formula reduces to the one given in the literature. However, as shown earlier, one does not need $\epsilon \ll 1$ to have inflation with DBI kinetic terms, so one may actually get more e-folds than potential domination suggests.

It is straightforward to write down corrections to observables related to the primordial perturbation spectra given the results of Garriga and Mukhanov [10], who have calculated the spectrum of perturbations for arbitrary kinetic terms to leading order in the Hubble slow roll parameters $\epsilon_{H}$ and

$$
\begin{aligned}
\eta_{H} & =-\frac{1}{H} \frac{d}{d t} \ln \epsilon_{H} \\
\sigma_{H} & =-\frac{1}{H} \frac{d}{d t} \ln c_{s} .
\end{aligned}
$$

The required smallness of $\eta_{H}$ and $\sigma_{H}$ expresses the condition that $\epsilon_{H}$ and the speed of sound $c_{s}$ should vary much more slowly than the scale factor. The results of [10] used below assume that $\epsilon_{H}, \eta_{H}, \sigma_{H}$ are small during the observable phase of inflation. Use of these formulae in the "ultra-relativistic" regime is also valid only under this assumption ${ }^{5}$.

The result for the primordial scalar power spectrum is 10

$$
P_{\mathcal{R}}=\frac{1}{36 \pi^{2} M_{P}^{4}} \frac{1}{c_{s}} \frac{\rho^{2}}{p+\rho},
$$

For DBI models one has [7] $c_{s}=1 / \gamma$. Here and in all the formulae below the right hand side is evaluated at the sound horizon exit $\left(c_{s} k=a H\right)$. In the slow roll expansion one finds

$$
P_{\mathcal{R}} \simeq \frac{1}{72 \pi^{2} M_{P}^{4}} \frac{V}{\epsilon_{V}}\left(1+\epsilon_{V} f V\right),
$$

while in the "ultra-relativistic" limit

$$
P_{\mathcal{R}} \simeq \frac{1}{36 \pi^{2} M_{P}^{4}}(1+\epsilon)^{2} f V^{2} .
$$

In models where $\epsilon \ll 1$ this is the same as found in [7, 29].

For the tensor perturbations one has [10]

$$
P_{h}=\frac{2}{\pi^{2} M_{P}^{2}} H^{2} .
$$

\footnotetext{
${ }^{5} \mathrm{An}$ interesting example where these formulae break down as a consequence of the slow roll conditions being violated is discussed in reference [13].
} 
This gives

$$
r \equiv \frac{P_{h}}{P_{\mathcal{R}}}=\frac{16 \epsilon_{H}}{\gamma},
$$

which in the slow roll approximation yields

$$
r \simeq 48 \epsilon_{V}\left(1-\epsilon_{V} f V\right) .
$$

For the spectral indices the results of Garriga and Mukhanov [10] can be written as follows:

$$
\begin{aligned}
n_{s}-1 & =-2 \epsilon_{H}+\eta_{H}+\sigma_{H} \\
n_{T} & =-2 \epsilon_{H} .
\end{aligned}
$$

These expressions are valid in the leading order in Hubble slow roll parameters. In the Hamilton-Jacobi formalism one finds

$$
\begin{aligned}
\eta_{H} & =\frac{4}{3 \gamma} \frac{H^{\prime \prime}}{H}-2 \epsilon_{H}+\sigma_{H} \\
\sigma_{H} & =-\frac{2}{3} \frac{H^{\prime}}{H} \frac{\gamma^{\prime}}{\gamma},
\end{aligned}
$$

which leads to the expression

$$
n_{s}-1=-4 \epsilon_{H}+2 \sigma_{H}+\frac{4}{3 \gamma} \frac{H^{\prime \prime}}{H} .
$$

Evaluating this to leading order in the slow roll expansion gives ${ }^{6}$

$$
n_{s}-1=-18 \epsilon_{V}+6 \eta_{V}+\frac{8}{27} \epsilon_{V} f^{\prime} V^{\prime} .
$$

The correction due to the DBI kinetic terms can be of either sign depending on the model.

\section{Conclusions}

Spacetime-filling $D$-branes moving on the 6-dimensional compact space appearing in a string compactification is a very natural setting for inflation in string theory.

\footnotetext{
${ }^{6}$ The unusual coefficients in this formula are a consequence of the adopted normalization of the potential slow roll parameters.
} 
While many structural aspects of this scenario are already known, many details which will be essential in determining whether this is how it happened are still unknown. Apart from questions about the precise ending of inflation, brane annihilation, energy transfer to standard model degrees of freedom and so on, there are still many technical aspects which need to be understood. This includes a reliable computation of the potential and the geometry of the throat in which the brane is moving while spacetime inflates. Existing examples are probably just the beginning. It is not even completely clear whether all the $D$-brane moduli effectively reduce to a single one relevant for driving inflation, as assumed in the class of effective field theories discussed here. While precise studies will clearly require numerical methods it is essential to explore approximate analytic tools in the regimes where some are available.

The main focus of this note was to look at the validity of the slow roll approximation for effective inflaton field theories with Dirac-Born-Infeld kinetic terms, which are are a rather distinctive feature of brane inflation. The slow roll expansion introduced in the case of canonical kinetic terms has a natural generalization to DBI models which can be computed easily using the iterative method presented here. It is obviously worthwhile to study such corrections in specific instances.

In the other extreme, that of large $\gamma$, is interesting that DBI models allow inflation with a higher ratio of kinetic to potential energy than is possible with canonical kinetic terms. This could significantly affect predictions at least in some cases. 


\section{References}

[1] G. R. Dvali and S. H. H. Tye, Phys. Lett. B 450, 72 (1999) [arXiv:hep-ph/9812483].

[2] F. Quevedo, Class. Quant. Grav. 19, 5721 (2002) [arXiv:hep-th/0210292].

[3] J. M. Cline, "String cosmology," arXiv:hep-th/0612129.

[4] R. Kallosh, "On Inflation in String Theory," arXiv:hep-th/0702059.

[5] S. H. Henry Tye, "Brane inflation: String theory viewed from the cosmos," arXiv:hep-th/0610221.

[6] E. Silverstein and D. Tong, Phys. Rev. D 70, 103505 (2004) [arXiv:hep-th/0310221].

[7] M. Alishahiha, E. Silverstein and D. Tong, Phys. Rev. D 70, 123505 (2004) [arXiv:hep-th/0404084].

[8] R. G. Leigh, Mod. Phys. Lett. A 4, 2767 (1989).

[9] C. Armendariz-Picon, T. Damour and V. F. Mukhanov, Phys. Lett. B 458, 209 (1999) [arXiv:hep-th/9904075].

[10] J. Garriga and V. F. Mukhanov, Phys. Lett. B 458, 219 (1999) [arXiv:hep-th/9904176].

[11] V. F. Mukhanov and A. Vikman, JCAP 0602, 004 (2006) [arXiv:astro-ph/0512066].

[12] A. Vikman, "Inflation with large gravitational waves," arXiv:astro-ph/0606033.

[13] G. Barenboim and W. H. Kinney, "Slow roll in simple non-canonical inflation," arXiv:astro-ph/0701343.

[14] S. Kachru, R. Kallosh, A. Linde, J. M. Maldacena, L. McAllister and S. P. Trivedi, JCAP 0310, 013 (2003) [arXiv:hep-th/0308055].

[15] H. Firouzjahi and S. H. Tye, JCAP 0503, 009 (2005) [arXiv:hep-th/0501099].

[16] X. Chen, Phys. Rev. D 71, 026008 (2005) [arXiv:hep-th/0406198].

[17] X. Chen, JHEP 0508, 045 (2005) [arXiv:hep-th/0501184].

[18] S. E. Shandera and S. H. Tye, JCAP 0605, 007 (2006) [arXiv:hep-th/0601099]. 
[19] S. Kecskemeti, J. Maiden, G. Shiu and B. Underwood, JHEP 0609, 076 (2006) [arXiv:hep-th/0605189].

[20] S. B. Giddings, S. Kachru and J. Polchinski, Phys. Rev. D 66, 106006 (2002) [arXiv:hep-th/0105097].

[21] J. E. Lidsey and D. Seery, Phys. Rev. D 75, 043505 (2007) [arXiv:astro-ph/0610398].

[22] D. Baumann and L. McAllister, "A microscopic limit on gravitational waves from D-brane inflation," arXiv:hep-th/0610285.

[23] P. J. Steinhardt and M. S. Turner, Phys. Rev. D 29, 2162 (1984).

[24] A. R. Liddle, P. Parsons and J. D. Barrow, Phys. Rev. D 50, 7222 (1994) [arXiv:astro-ph/9408015].

[25] L. P. Grishchuk and Yu. V. Sidorov in M. A. Markov, V. A. Berezin and V. P. Frolov, "Quantum Gravity. Proceedings, 4TH Seminar, Moscow, USSR, May 25-29, 1987", World Scientiic (1988).

[26] A. G. Muslimov, Class. Quant. Grav. 7, 231 (1990).

[27] D. S. Salopek and J. R. Bond, Phys. Rev. D 42, 3936 (1990).

[28] W. H. Kinney, Phys. Rev. D 56, 2002 (1997) [arXiv:hep-ph/9702427].

[29] X. Chen, M. x. Huang, S. Kachru and G. Shiu, JCAP 0701, 002 (2007) [arXiv:hep-th/0605045]. 